Review

\title{
Classification of Solitary Plasmacytoma, Is it more Intricate than Presently Suggested? A Commentary
}

\author{
Nissim Ohana $1^{*}$, Ory Rouvio ${ }^{2}$, Karen Nalbandyan ${ }^{3}$, Dimitri Sheinis ${ }^{1}$, Daniel Benharroch ${ }^{\natural}$ \\ 1. Orthopedic Surgery, Soroka University Medical Center and Faculty of Health Sciences, Ben Gurion University of the Negev, Beer-Sheva, Israel. Present \\ Address: Orthopaedics, Meir Medical Center, Kfar-Saba, Affiliated to Tel-Aviv University, Israel. \\ 2. Hematology Division, Soroka University Medical Center and Faculty of Health Sciences, Ben Gurion University of the Negev, Beer-Sheva, Israel. \\ 3. Hematopathology Service, Pathology Department, Soroka Univ. Med. Ctr. and Faculty of Health Sciences, Ben Gurion University of the Negev, Beer-Sheva, \\ Israel. \\ * Similar contribution. \\ $\square$ Corresponding author: Prof. Daniel Benharroch, Independent Physician, Pathology Department, Soroka University Medical Center, 1, Rager Blvd, P.O.Box \\ 151, Beer-Sheva, Israel. Tel. +972-507579140; Fax. -86232770. e-mail: danielbenharroch1@gmail.com \\ (c) Ivyspring International Publisher. This is an open access article distributed under the terms of the Creative Commons Attribution (CC BY-NC) license \\ (https:// creativecommons.org/licenses/by-nc/4.0/). See http://ivyspring.com/terms for full terms and conditions.
}

Received: 2018.04.24; Accepted: 2018.08.28; Published: 2018.10.10

\begin{abstract}
A query regarding the definition and the classification of solitary plasmacytoma is apparently still pending. The clinical course, the response to treatment and the propensity to progress to plasma cell myeloma, are all a function of the classification which must be established on a firm basis. Solitary plasmacytoma should be recognized in the continuum of the plasma cell neoplasms. Moreover, whether the solitary plasmacytoma of bone and the extramedullary type of the tumor represent two distinct disease entities, exhibiting separate biological characteristics, has not been finally established. To appraise the similarities and differences between these two types of lesion, we have scrutinized recent investigations relating their classification. A commentary highlighting our conclusions follows.
\end{abstract}

Key words: solitary plasmacytoma; solitary plasmacytoma of bone; occult bone marrow disease

\section{Introduction}

Among the plasma cell neoplasms, the least frequent $(5 \%$ or less) represents a type of lesion, isolated in bone, most often in the axial skeleton, or in soft tissue, mainly in the head and neck mucosae. They are composed of clonal plasma cells and showing a variable tendency to progress to plasma cell myeloma (PCM, multiple myeloma, MM). This condition has never before been considered literally as a precursor of myeloma. Moreover, controversy is still pending, relating the similarities and discrepancies which prevail between the solitary plasmacytoma of bone (SPB), considered the most frequent and especially apt to transform into $\mathrm{MM}$, and the extramedullary plasmacytoma (EMP). To clarify criteria, definitions and classification, we present a commentary evolving around several of the pertinent studies published in the last several years.

\section{Solitary Plasmacytoma}

Solitary plasmacytoma (SP) is a clonal disorder of plasma cell origin, most frequently limited to one site of the axial skeleton or to a soft tissue area, mainly of the upper respiratory tract. The solitary plasmacytoma of bone, is considered to present a marked propensity to progress into multiple myeloma (MM), when compared to the second lesion, the solitary extramedullary plasmacytoma. It is widely agreed that the two conditions are indeed distinct entities (McKenna et al [1, 2]).

The purpose of this commentary is to scrutinize the break-up of these two lesions and to bring some clarity into the sub-classification. Moreover, in some patients an additional, but minimal clonal plasmacytosis is disclosed in the bone marrow, remote from the SP. 
For all forms of SP, radiotherapy is the first line of treatment. Some oncologists will extend their care by using surgery. Adjuvant chemotherapy has rarely been offered as an additional or unique modality of treatment $[1,2]$.

Although a distinction is clearly made between SPB and EMP, one can identify the mark of the International Myeloma Working Group (IMWG) updated criteria for the diagnosis of multiple myeloma in the two tables published by McKenna et al, although very discreetly [1-3]. Moreover, McKenna et al stated that EMP is biologically separate from SPB and PCM [1]. In the updated, 4th Edition of the WHO Classification of Tumours of Haematopoietic and Lymphoid Tissues [2], these two lesions are presented as two distinct types of tumors.

Furthermore, the WHO update describes the SPB as being composed of two subtypes: a SPB which lacks clonal marrow plasma cells remote from the plasmacytoma itself and thus, discloses a normal bone marrow; it may progress to MM in three years, in $10 \%$ of cases, whereas the other SPB subtype exhibits minimal $(<10 \%)$ clonal bone marrow plasma cells [2]. The progression rate of this second subtype of SPB to PCM reaches $60 \%$ in 3 years. A similar dichotomy regarding the EMP is not delineated in this update. Otherwise, SPB and EMP are clearly distinguished one from the other, with no overlap demonstrated $[1,2]$.

When scrutinizing the International Myeloma Working group (IMWG) Updated Criteria for the Diagnosis of Multiple Myeloma [3], a previous study cited in the above mentioned reports, a somewhat more complicated picture is disclosed. The sharp delineation between SPB and EMP as described in the WHO update document, is not adopted with the IMWG conclusions. In this last report, the two isolated plasmacytoma types blend into a single solitary plasmacytoma [3], which we shall describe as "not otherwise specified" (NOS). This is believed to represent a single type lesion, either of bone or of soft tissue composed of clonal plasma cells of variable differentiation. In these patients, the iliac crest bone marrow shows no clonal plasma cells at diagnosis. When excluding the isolated primary tumor, the spine and pelvis disclose only normal bones by MRI or by $\mathrm{CT}$. Moreover, no end-organ damage, related directly to the plasma cell disorder is evident, like hypercalcemia, renal insufficiency, anemia or bone injury (CRAB). About $10 \%$ of these patients might advance to $\mathrm{MM}$ within 3 years [3].

The IMWG study further segregates from the above group, solitary plasmacytomas with minimal $(<10 \%)$ clonal bone marrow plasma cells (BMPC) at diagnosis. This subtype differs from the so called
SP-NOS, by one feature only: evidence of $<10 \%$ clonal BMPC at diagnosis. But, the course of this subset contrasts markedly: in the SPB of this subtype, $60 \%$ of cases will advance to PMC (MM) within 3 years. In contrast, the EMP of this subset will progress to PCM in 3 years, in $20 \%$ of cases only [3] (Table 1 ).

Table 1. Definition and course of solitary plasmacytoma (Adapted from [3]).

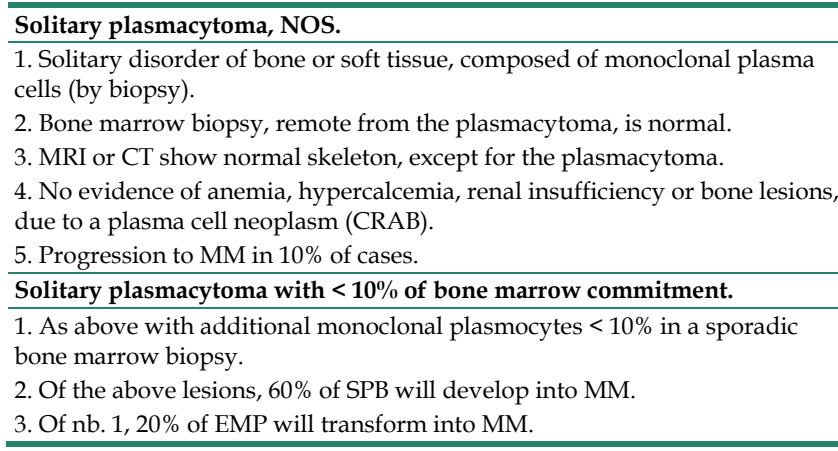

For the sake of simplicity, we will not discuss in this commentary, the rate of recurrence of SP which may reach $25 \%$, nor the development of multiple SPs, evaluated at about $30 \%$, including their differential diagnosis from PCM.

The above classification of SP, which we have compiled from the IMWG report [3], varies from that of the WHO 4th edition update [2]. Moreover, this dissimilarity may account for Pavia et al (in their Table 1) disclosing only a small proportion of statistically significant variance between SPB and EMP [4]. It is noteworthy, that many of the patients who progress to PCM, will demonstrate an indolent form of the myeloma [2].

The SP definition and classification presented in the WHO update and in additional articles [2, 4-6], consider the divergence of SPB from EMP, to be the basis of their biologic differences. By assessing the more stringent significance of the IMWG class system, we suggest a more complex classification (the sub-type SP-NOS has been included by our commentary) and the inclusion of at least, three pathogenic pathways in accounting for the similarities and the discrepancies in the various forms of solitary plasmacytoma. The first is discussed in a few investigations and concerns the interactions between the SPs and their bone marrow microenvironment, as follows. This might be critical, notably in the early stages of myelomagenesis [6]. The involvement of clonal plasma cells with the bone marrow environment may determine also the stage of the SP [7]. More insight may be obtained from similar studies on the PCM with its surroundings [8-12]. 
The interaction of the clonal plasma cells with their background is also relevant in extramedullary locations. The EMP was investigated, among other plasma cell neoplasms, for the expression profiles of several markers. They revealed an increased pattern of angiogenesis, notably related with $\mathrm{NOTCH} 3$, CD31, angiopoietin 1 and fibronectin 1 expression [13].

The second pathogenic pathway relates to the "occult marrow disease". The clonal BMPC represent a minority at diagnosis and might be inaccessible and therefore, under-diagnosed by classical imaging and be minimized by bone marrow biopsy immunophenotyping. However, modern diagnostic methodology, includes the evaluation of the BMPC which should be routinely performed by multiparameter flow cytometry, serum and/or urine free light chain ratio $[5,6,14]$.

Plasma cell-related bone disease, the third pathogenic process, might be detected more precisely, using low-dose whole-body $\mathrm{CT}$, the MRI, the FDG-PET and PET-CT. Regarding the SPB, bone destruction was analyzed, including an increased osteoclastic resorption, followed by decreased bone formation; the osteoclastic resorption being a very early event, occurring long before the first symptoms of the disease. Inflammatory cytokines, osteoclast activator factors, and osteoclast colony stimulating factors, originate in tumor plasma cells, as well as from the hematopoietic microenvironment [15].

Thus, as the imaging and laboratory exams become more and more accurate, and an occult marrow disease is more widely recognized [16, 17], the diagnosis of SP might be altered into one of the types of PCM [6]. Similarly, more patients will be diagnosed as suffering of PCM, when a more precise diagnosis of renal insufficiency is established by creatinine clearance analysis, instead of serum creatinine levels only. These modern technical amends might account for the alteration in the incidence of SP from about 5\% SPB and 5\% EMP, to as little as 1-2\% SPB with 1\% EMP [2]. One may assume that the remainder has been identified as one form or other of MM.

\section{Conclusions}

The main findings highlighted by the present commentary concern the disparity exhibited by the sub-classification of the SP, between those suggested by McKenna et al $[1,2]$ on the one end and that presented by Rajkumar et al [3]. The novelty of our position lays in our adoption of the medical attitude on this issue, in contrast with that of the pathologists stance. In the case our point of view was adopted, and considering the rare occurrence of the SP, the impact might be limited. However, the lesion described in this commentary as solitary plasmacytoma, NOS, might be conferred a "wait-and-see" attitude, providing however repeated bone marrow biopsies.

An isolated study has stated that no essential variance was to be found between SPB and EMP, but it was published a very long time ago [18].

Inconsistencies have been found between the criteria and the classification of SP, as they appear in the IMWG report and in other articles. It is notable that the IMWG study concerns multiple myeloma as well as the other related plasma cell neoplasms [3]. Thus, when the WHO update document addresses the SP types separately, some of the principles delimited by the definition and classification of SP in the IMWG paper [3], might be lost, even though the outlines are mentioned exclusively in tables, in both of McKenna et al discussions [1, 2]. In addition, there seems to be a common denominator to both SPB and EMP, representing the SP-NOS group, which is not highlighted in the WHO document [2]. The IMWG report lumps together SPB and EMP at baseline, with a $10 \%$ progression potential only; whereas minimal clonal marrow plasma cells $(<10 \%)$ found at diagnosis, will dictate a more severe clinical course for SPB (60\% progression to PCM), and to a lesser degree for EMP as well (20\% progression to PCM) [3].

The pathogenic contribution of the BMPC on the one hand, and of the bone (marrow) microenvironment, in contrast with the soft tissue milieu, on the other hand, are the putative factors that will turn a homogeneous lesion (SP-NOS), into a heterogeneous group of disorders with variable clinical progression to PCM.

\section{Acknowledgements}

We thank Kibbutz Sde-Boker for their support.

\section{Authorship}

N.O. Initiated the idea; confirmed the first draft; reviewed the last version. O.R. Sustained the project; approved the first draft; reviewed the last version. K.N. Contributed the bibliography; reviewed the last version. D.S. Contributed his experience as a surgeon; reviewed the last version. D.B. Sustained the project; wrote the first draft and integrated the revisions; approved the last version.

\section{Competing Interests}

The authors have declared that no competing interest exists.

\section{References}

1. McKenna RW, Kroft SH, Linden MA. Solitary plasmacytoma. In: Jaffe ES, Arber DA, Campo E, Harris NL, Quintanilla-Martinez L, eds. Hematopathology, 2nd ed. Philadelphia: Elsevier; 2017: 496-498. 
2. McKenna RW, Kyle RA, Kuehl WM, Harris NL, Coupland RW, Fend F. Plasmacytoma. In: Swerdlow SH, Campo E, Harris NL, Jaffe ES, Pileri SA, Stein $\mathrm{H}$, Thiele J, eds. WHO Classification of Tumours of Haematopoietic and Lymphoid Tissues, 4th edit update, Lyon, IARC, 2017: 250-253.

3. Rajkumar SV, Dimopoulos MA, Palumbo A, et al. International Myeloma Working Group Updated Criteria for the Diagnosis of Multiple Myeloma. Lancet Oncol. 2014; 15: e538-548.

4. Pavia B, Chandia M, Vidriales M-B, et al. Multiparameter flow cytometry for staging of solitary bone plasmacytoma: new criteria for risk of progression to myeloma. Blood. 2014; 124: 1300-1303.

5. Hill QA, Rawstron AC, de Tute RM, Owen RG. Outcome prediction in plasmacytoma of bone: a risk model utilizing bone marrow flow cytometry and light chain analysis. Blood. 2014; 124: 1296-1299.

6. Warsame R, Gertz MA, Lacy MQ et al. Trends and outcomes of modern staging of solitary plasmacytoma of bone. Am J Hematol. 2012; 87: 647-651.

7. Korde N, Maric I. Myelomagenesis: capturing microenvironment changes. Sem Hemato. 2011; 48: 13-21.

8. Storti P, Marchica V, Giuliani N. Role of galectins in multiple myeloma. Int J Mol Sci. 2017; 18. pii: E2740.

9. Ring ES, Lawson MA, Snowden JA, Jolley I, Chantry AD. New agents in the treatment of myeloma bone disease. Calcif Tissue Int. 2017 [Epub ahead of print].

10. Musolino C, Allegra A, Innano V, Allegra AG, Pioggia G, Gangemi S. Inflammatory and anti-inflammatory equilibrium, proliferative and anti-proliferative balance: the role of cytokines in multiple myeloma. Mediators Inflamm. 2017; 2017: 1852517.

11. Manni S, Carrino M, Piazza F. Role of protein kinases CK1a and CK2 in multiple myeloma: regulation of pivotal survival and stress managing pathways. J Hematol Oncol. 2017; 10: 157.

12. Moschetta M, Kawano Y, Sacco A, et al. Bone marrow stroma and vascular contributions to myeloma bone homing. Current Osteoporos Rep. 2017; 15: 499-506.

13. Hedvat $\mathrm{CV}$, Comenzo RL, Teruya-Feldstein J, et al. Insights into extramedullary tumor cell growth revealed by expression profiling of human plasmacytomas and multiple myeloma. Br J Haematol. 2003; 122: 728-744.

14. Dingli D, Kyle RA, Rajkumar SV, et al. Immunoglobulin free light chains and solitary plasmacytoma of bone. Blood. 2006; 108: 1979-1983.

15. Bataille R. The mechanism of bone lesions in human plasmacytomas. Stem cells. 1995; 13 Suppl 2: 40-47.

16. Fouquet G, Guidez S, Herbaux C, et al. Impact of initial FDG - PET/CT and serum-free light chains on transformation of conventionally defined solitary plasmacytoma to multiple myeloma. Clinical Cancer Res. 2014; 20: 3254-3260.

17. Bink K, Haralambieva E, Kremer $M$, et al. Primary extramedullary plasmacytoma: similarities with and differences from multiple myeloma revealed by interphase cytogenetics. Hematologica. 2008; 93: 623-626.

18. Meis JM, Butler JJ, Osborne BM, Ordonez NG. Solitary plasmacytomas of bone and extramedullary plasmacytomas. Cancer. 1987; 59: 1475-1485.

19. Finsinger P, Grammatico S, Chisini M, Piiocchi A, Foa R, Petrucci MT. Clinical features and prognostic factors in solitary plasmacytoma. Br J Haematol. 2016; 172: $554-560$.

20. Reed V, Shah J, Medeiros LJ, et al. Solitary plasmacytoma outcome and prognostic factors after definitive radiation therapy. Cancer. 2011; 117: $4468-4474$ 